\title{
Analysis on Deformation Failure and Structural Mechanical Characteristics of Multistage Loess Slope Supported by Frame Structure with Anchors
}

\author{
Kui-bin Yang $\mathbb{D}^{1,2,3}$ Yan-peng Zhu, ${ }^{1,2,3}$ Lin-ping Wu, ${ }^{1,2,3}$ Xin-guo Duan, ${ }^{1,2,3}$ \\ Duo-bang Shi, ${ }^{1,2,3}$ and Xiao-tao $\mathrm{Du}^{1,2,3}$ \\ ${ }^{1}$ School of Civil Engineering, Lanzhou University of Technology, Lanzhou 730050, China \\ ${ }^{2}$ Key Laboratory of Disaster Mitigation in Civil Engineering of Gansu Province, Lanzhou University of Technology, \\ Lanzhou 730050, China \\ ${ }^{3}$ Western Engineer Research Center of Ministry of Education for Disaster Mitigation in Civil Engineering, Ministry of Education, \\ Lanzhou University of Technology, Lanzhou 730050, China \\ Correspondence should be addressed to Kui-bin Yang; ykbin93@163.com
}

Received 18 November 2021; Accepted 4 January 2022; Published 18 January 2022

Academic Editor: Jiang Hu

Copyright (C) 2022 Kui-bin Yang et al. This is an open access article distributed under the Creative Commons Attribution License, which permits unrestricted use, distribution, and reproduction in any medium, provided the original work is properly cited.

\begin{abstract}
Frame anchor supporting structure is widely accepted in the loess area, especially in the multistage slopes along the road. However, the development between theory and practice is not synchronous, and there exist experience and uncertainty in the design process to a great extent. In response to this problem, the multistage loess slope supported by frame structure with anchors is taken as the research object, and the model test is carried out with the help of the large-scale geotechnical centrifuge under the condition of using prototype materials as much as possible. Then, according to the test data and phenomena, the structural stress and slope deformation concerned in the project are analyzed. The main differences in stress and deformation between the multistage loess slope supported by frame structure with anchors and other types of slopes are pointed out, and some targeted suggestions are given. The research results show that the frame anchor supporting structure and soil can always maintain coordinated deformation during centrifugal loading, and the failure process has certain toughness characteristics. The stress characteristics of the anchor rod and the sliding deformation range of the slope in the multistage loess slope supported by frame structure with anchors are different from the slope with structural surface and single-stage slope. The length of the anchoring section cannot be completely determined based on the potential sliding surface in the design. It is reasonable to strictly control the end position of the anchoring section from the perspective of changing the position of the potential sliding surface. The results obtained can enrich and improve the stability theory of multistage loess slope and provide guidance for engineering practice.
\end{abstract}

\section{Introduction}

In recent years, with the continuous improvement of the scale of transportation infrastructure, a large number of high and steep slope projects have appeared in the loess area $[1,2]$. In order to ensure the safety of the slope, the high and steep slopes are often graded in practical projects to form a stepped multistage slope, and support measures are used for reinforcement. As a common type of support structure, the frame anchor supporting structure has a good support effect and has been widely used in the multistage slopes of the loess area $[3,4]$. However, as far as the current application is concerned, the development of theory and practice in multistage loess slope supported by frame structure with anchors is not synchronous. There is a certain degree of conservativeness and blindness when adopting support measures. The waste of resources caused by excessive support and the potential safety hazards caused by insufficient support always exist, and instability accidents often occur. Therefore, it is not only the need for theoretical development but also the urgent requirement of engineering construction to carry out the research on the related problems of 
multistage loess slope supported by frame structure with anchors.

For slopes supported by frame structure with anchors, many scholars have studied the stability calculation through theoretical analysis and given the calculation formula of the safety factor and the search method of the potential sliding surface under different conditions [5-8]. However, only theoretical analysis is not enough, and the solution of engineering problems still needs to be considered from the perspective of the mechanism. At present, the research on the stress of support structure and the deformation characteristics of the slope is an effective way to understand the mechanism of slope instability, and there are many corresponding research methods. Numerical simulation can not only generate the failure information automatically in the analysis process, reflect the gradual instability of the slope [9], but also obtain the mechanical behavior of the support structure and the slope response under different working conditions [10-12]. However, the constitutive relationship of soil is complex, and the reliability of parameters is difficult to master. Moreover, the basis for judging instability is not unique. There are still different opinions on which criterion to use, and a unified standard has not been formed, and many problems need to be further deepened $[13,14]$. The insitu monitoring is the most intuitive and most in line with the actual situation, but this method has a complex operation and high cost. It has only been applied in a few engineering constructions, and the corresponding research results are few [15]. Although the ordinary physical model test is simple and intuitive, the stress level of the model is not similar to that of the prototype. It is difficult to truly reflect the stress variation law of the prototype in slope engineering with self-weight stress as the dominant factor [16-19]. The centrifugal test uses centrifugal acceleration to compensate for the weight loss of the scaled model. It can not only reflect the real stress state of the prototype slope but also shows the deformation behavior and failure process similar to the prototype [20, 21]. With the aid of centrifugal model tests, researches on soil nail reinforced slopes, geotextile reinforced slopes, and antislide pile reinforced slopes can all achieve good results [22-29]. However, the load of most centrifugal test equipment is limited and the size of the model box is small $[30,31]$, so there are few centrifugal model tests that can reflect the real stress state of multilevel support slope. The experimental research of supporting slope mainly focuses on field test, general static model test, and shaking table model test [32-35]. It can be seen that experts and scholars have conducted a lot of research on the slopes supported by frame structure with anchors from the aspects of theoretical analysis, experimental research, and numerical simulation and have obtained many meaningful conclusions. However, these studies cannot fully meet the needs of the development of engineering practice. On the one hand, the research objects are mostly single-stage slopes, and there are relatively few studies on the multistage slopes widely distributed along highways and railways, especially the joint effect of multistage frame anchor supporting structures. On the other hand, slope instability caused by unreasonable design and construction occurs from time to time, but there are few analyses on the deformation characteristics of loess slopes supported by frame anchors.

In view of the shortcomings of the above-mentioned existing research, this paper takes the three-stage loess slope supported by frame structure with anchors as the research object, selects the prototype material for small-scale model making as far as possible, and carries out the model test with the help of large geotechnical centrifuge. Through the analysis of test data and test phenomena, the deformation and failure characteristics of multistage loess slope and the stress distribution law of frame anchor supporting structure are obtained. At the same time, according to the stress state of the anchor rod and the deformation range of the slope, the main differences between the multistage loess slope and the slope with the structural plane and single-stage slope under the condition of frame anchor support are pointed out, and some targeted suggestions are given for the frame anchor support of multistage loess slope.

\section{Centrifugal Model Test Design}

2.1. Main Parameters of Prototype Slope. The highway slope has three levels. Each level is $8 \mathrm{~m}$ high, the total height is $24 \mathrm{~m}$, and the slope rate of each level is $1: 0.7$. Frame anchor structure is used for support in design. From top to bottom, the length of nonanchored section of the anchor rod is $6 \mathrm{~m}$, $5 \mathrm{~m}$, and $5 \mathrm{~m}$, respectively, and the length of the anchored section is $10 \mathrm{~m}, 9 \mathrm{~m}$, and $7 \mathrm{~m}$, respectively. The horizontal and vertical spacing of anchor rods is $2.5 \mathrm{~m}$, and the overall effect is shown in Figure 1. The geological conditions are composed of loess and sandstone, in which the slope body is loess and the underlying stratum is sandstone. The soil layer parameters are obtained from the quick shear test, and the specific values are shown in Table 1 .

\subsection{Test Equipment and Similarity Ratio Determination.}

The similarity ratio of materials in the centrifugal model test is $1: 1$, so materials similar to the prototype should be selected as far as possible. However, the multistage slope supported by frame structure with anchors involves two structures: frame and anchor rod, which are very small in size relative to the slope. In the manufacturing process, if the size of the similarity model is large, the existing centrifugal equipment cannot meet the test requirements, and if the size of the similarity model is small, the supporting structure is difficult to make.

In order to solve the contradiction between the scale ratio and the fabrication of supporting structures, it is necessary to control the size of the model within a reasonable range and select a large geotechnical centrifuge to meet the test requirements. After further investigation and comparison, the TLJ-500 geotechnical centrifuge of the State Key Laboratory of Geological Disaster Prevention and Control of the Chengdu University of Technology is used in the test, and the specific model box size is $1.0 \mathrm{~m} \times 0.6 \mathrm{~m} \times 0.8 \mathrm{~m}$. The corresponding geotechnical centrifuge parameters are shown in Table 2, and the model similarity ratio is shown in Table 3. 


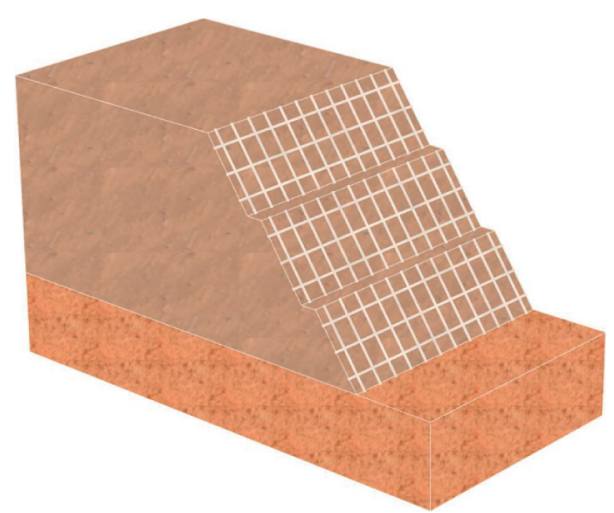

FIGURE 1: Prototype of the three-stage loess slope supported by the frame structure with anchors.

TABle 1: Physical and mechanical parameters of soil.

\begin{tabular}{lccc}
\hline Stratum & $\gamma\left(\mathrm{kN} / \mathrm{m}^{3}\right)$ & $c(\mathrm{kPA})$ & $\varphi(\circ)$ \\
\hline Loess & 16.5 & 15 & 25 \\
Sandstone & 22 & 150 & 35 \\
\hline
\end{tabular}

TABLE 2: Main technical indexes of the geotechnical centrifuge.

\begin{tabular}{lccccc}
\hline $\begin{array}{l}\text { Centrifuge capacity } \\
(\mathrm{g} \cdot \mathrm{t})\end{array}$ & $\begin{array}{c}\text { Centrifuge radius } \\
(\mathrm{m})\end{array}$ & $\begin{array}{c}\text { Effective radius } \\
(\mathrm{m})\end{array}$ & $\begin{array}{c}\text { Centrifugal acceleration } \\
(\mathrm{g})\end{array}$ & $\begin{array}{c}\text { Speed stability }(\% \mathrm{FS} / \\
12 \mathrm{~h})\end{array}$ & $\begin{array}{c}\text { Model box size } \\
(\mathrm{m} \times \mathrm{m} \times \mathrm{m})\end{array}$ \\
\hline 500 & 4.5 & 4 & $10-250$ & 0.5 & $1.0 \times 0.6 \times 0.8$ \\
\hline
\end{tabular}

TABLE 3: Similarity ratios of the constant stress centrifugal model tests.

\begin{tabular}{lccc}
\hline Physical quantity & Similarity ratio & Physical quantity & Similarity ratio \\
\hline Linear displacement & $1 / n$ & Internal friction angle & 1 \\
Area & $1 / n^{2}$ & Cohesion & 1 \\
Volume & $1 / n^{3}$ & Stress & 1 \\
Density & 1 & Strain & 1 \\
Moisture content & 1 & Bending moment & $1 / n^{3}$ \\
Bulk density & $1 / n$ & Time & $1 / n^{4}$ \\
\hline
\end{tabular}

The similarity ratio of this test is determined as $50: 1$. The height of the model slope is $480 \mathrm{~mm}$, the sandstone layer is considered as $200 \mathrm{~mm}$, the corresponding total filling height is $680 \mathrm{~mm}$, the distance between the edge of the slope top and the inner wall on the left side of the model box is $434 \mathrm{~mm}$, and the distance between the slope toe and the inner wall on the right side of the model box is $150 \mathrm{~mm}$. The specific dimensions are shown in Figure 2.

2.3. Similar Material Production of the Model. In order to restore the structural stress and slope deformation characteristics under the real stress state as much as possible, the model soil and supporting structure are prepared with materials similar to the prototype. Taking the physical and mechanical parameters of the actual soil as the standard, the loess from the site is remolded by controlling the water content and bulk density, and the remolded loess with similar properties to the undisturbed loess is obtained, in which the target water content is $9.5 \%$. Sandstone is formed by mixing crushed and screened loess and cement in the mass ratio of 5:1 and adding water, in which the target moisture content is $8.0 \%$. The supporting structure is made of cement slurry and galvanized iron wire. The beam and column of the frame belong to flexural members in practical work, and the similarity ratio between the maximum bending moment and the prototype meets Table 3 . The section size is $8 \mathrm{~mm} \times 10 \mathrm{~m}$, and two galvanized iron wires with a diameter of $0.72 \mathrm{~mm}$ are selected for reinforcement, in which the yield strength of galvanized iron wire is $195 \mathrm{MPa}$ and the cement strength is C30. The anchor rod is mainly elastic deformation. Considering the axial tension of the rod core reinforcement, the diameter of the anchoring section, and the manufacturing difficulty, the galvanized iron wire with a diameter of $1 \mathrm{~mm}$ is finally selected as the similar material of the rod core, and the diameter of the anchoring section is $8 \mathrm{~mm}$. At the same time, small wire clamping appliances are used as connecting members and poured together with the frame structure. This can ensure that the galvanized iron wire is firmly connected with the frame and avoid the damage of the anchor head connection under high centrifugal acceleration. The frame and anchor rod after fabrication are shown in Figure 3. 


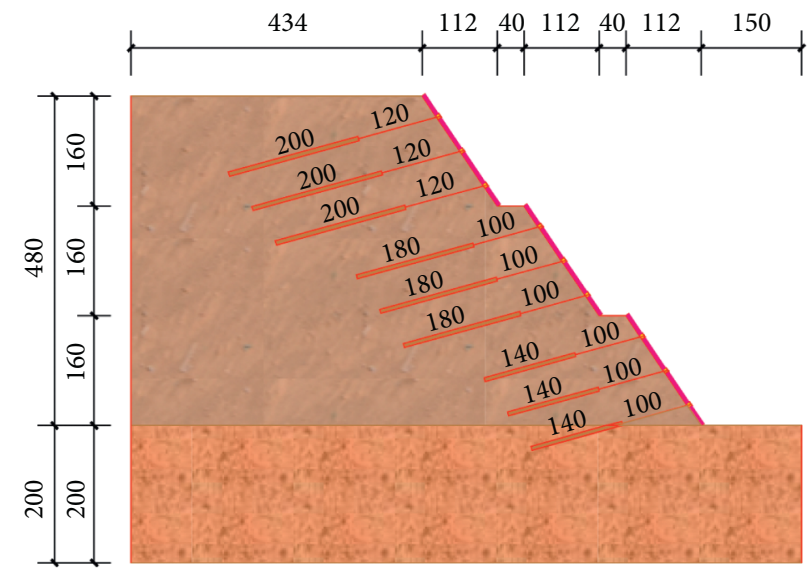

FIgURE 2: Dimensions of the centrifugal model slope (unit: $\mathrm{mm}$ ).

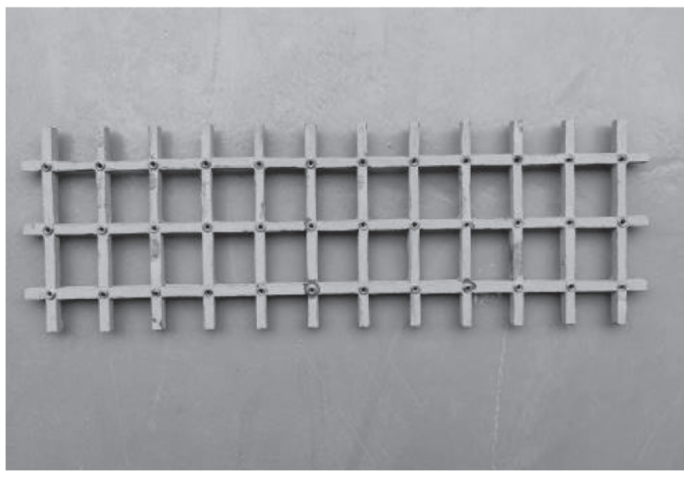

(a)

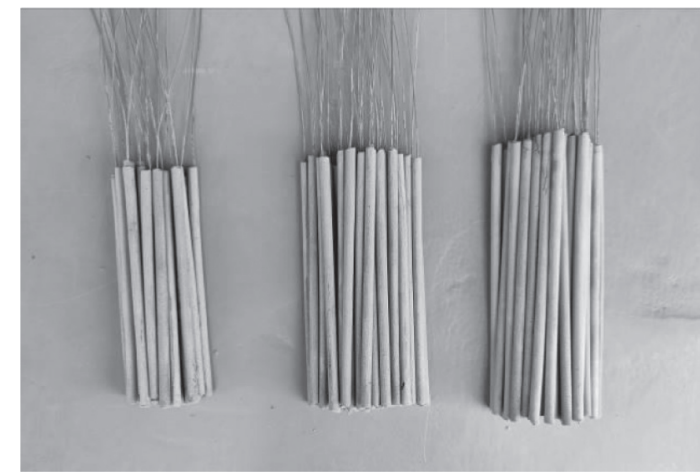

(b)

FIgure 3: Fabrication of frame anchor structure. (a) Model frame. (b) Model anchor.

2.4. Sensor Arrangement of the Supporting Structure. In order to obtain the distribution of slope stress, Earth pressure on supporting structure and axial force of anchor rod during centrifugal loading, Earth pressure sensors are embedded in the slope and under the frame column, and strain gauges are pasted on the anchoring section of anchor rod. There are 8 Earth pressure sensors in total. T1, T2, T3, T4, and T5 are buried inside the slope, and T6, T7, and T8 are buried below the frame column. The relative position is shown in Figure 4. There are three anchor rods tested, which are located in the middle row of the middle column of each grade of the slope. Five strain monitoring points are evenly and symmetrically arranged in the anchoring section of each anchor rod, as shown in Figure 5.

2.5. Model Slope Making Process. Firstly, draw the slope shape line on the sidewall of the model box to facilitate model filling and apply silicone oil to reduce the boundary effect caused by sidewall friction. Then, the soil is weighed and filled in layers. After filling to the preset position of each layer of anchor rod, the filled soil shall be locally cut to provide an inclined plane with an inclination of $15^{\circ}$ for the arrangement of the anchor rod, and the soil shall be covered and compacted after the anchor rod is placed. After filling to the slope top elevation, the soil shall be excavated according to the design slope rate. The excavation is divided into three stages, each of which is $160 \mathrm{~mm}$. Support shall be carried out immediately after each level of excavation, and the edge of the slope shall not be damaged during operation. When placing the frame, the galvanized iron wire passes through the anchor holes in sequence, and the final frame is close to the slope surface. Finally, in order to observe the deformation development law of the slope during centrifugal loading, small pushpins with a spacing of $50 \mathrm{~mm}$ are set on the slope. The slope model after fabrication is shown in Figures 6 and 7 .

2.6. Test Loading Process. Firstly, the model slope is placed for one week before loading to complete the initial consolidation. Secondly, in order to consider the further consolidation of the slope during loading, the loading process is carried out according to two frequencies. At the beginning, the centrifugal acceleration increased by $10 \mathrm{~g}$ each time for 2 min. After the centrifugal acceleration exceeds $50 \mathrm{~g}$, increase $5 \mathrm{~g}$ each time, and the stabilization time increases to $5 \mathrm{~min}$. The final acceleration reaches $80 \mathrm{~g}$. 


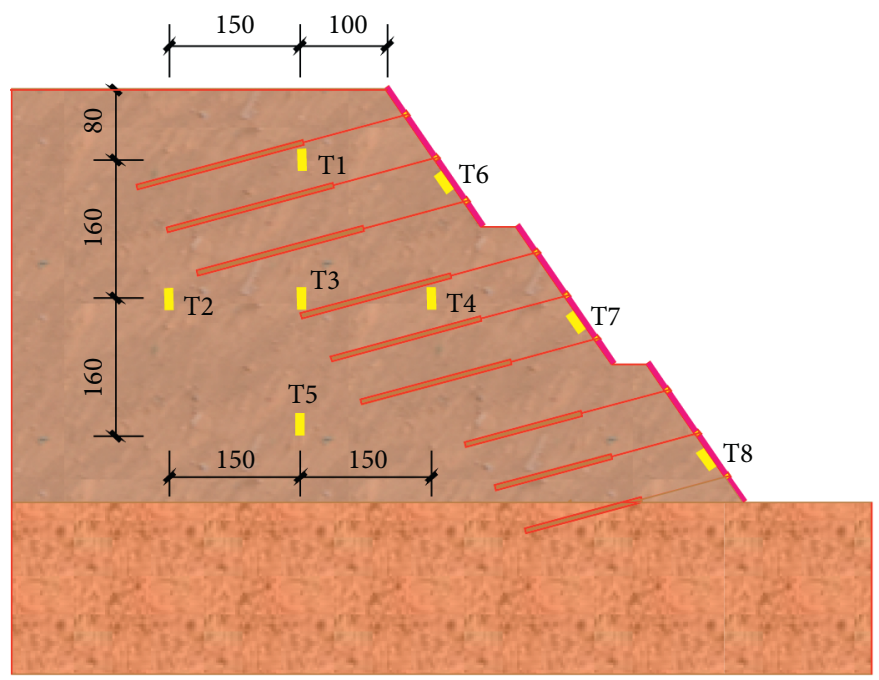

Figure 4: Arrangement of Earth pressure sensors (unit: mm).

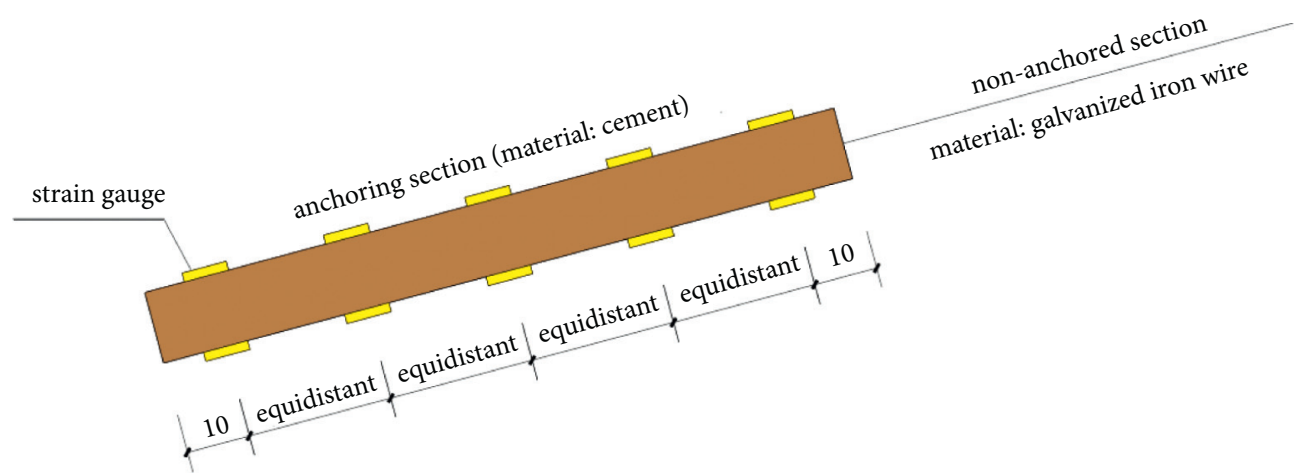

Figure 5: Arrangement of strain gauges on the anchor rod (unit: $\mathrm{mm}$ ).

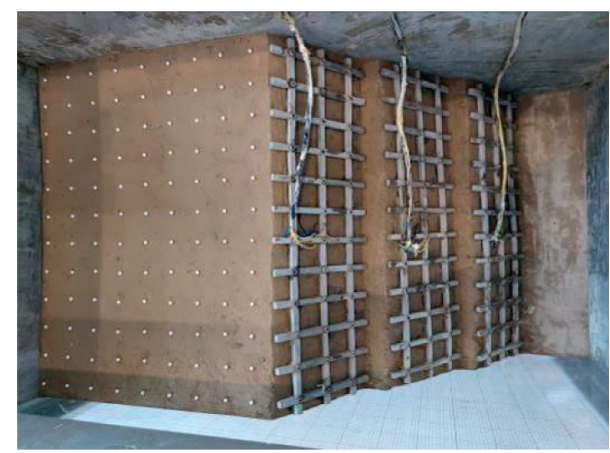

Figure 6: Arrangement of slope displacement monitoring points.

\section{Analysis of Centrifugal Test Results}

3.1. Analysis of Deformation and Failure Characteristics during Loading. On the whole, the frame anchor supporting structure has a good support effect on the multistage loess slope. Although the slope is deformed under high centrifugal acceleration, there is no obvious overall collapse. The structure and soil can always maintain coordinated deformation, and the failure process has certain toughness characteristics.
It can be seen from the loading process that with the gradual increase of the loading amount, the slope deformation becomes more and more obvious, but there are some differences in the deformation in different regions, and the slope deformation mode is also different under different accelerations. When the loading amount is small, the deformation is mainly vertical settlement, the horizontal displacement is very small, and the consolidation settlement occurs on the whole slope. When the acceleration exceeds $20 \mathrm{~g}$, the vertical settlement continues to increase and the horizontal displacement begins to appear. This is because the existence of the slope surface makes the soil appear with lateral displacement while further consolidating. When the acceleration exceeds $60 \mathrm{~g}$, the uneven settlement of each area on the slope top becomes more and more obvious, the vertical settlement near the slope surface side is relatively large, and the transverse cracks begin to appear at the rear edge of the slope top. With the continuous increase of acceleration, the soil settlement rate behind the crack decreases gradually, the soil settlement rate in front of the crack increases gradually, and the crack increases continuously. Finally, the cracks at the rear edge of the slope top are penetrated. The high-resolution images of the centrifuge and the deformation of the slope are shown in Figures 8 and 9. 


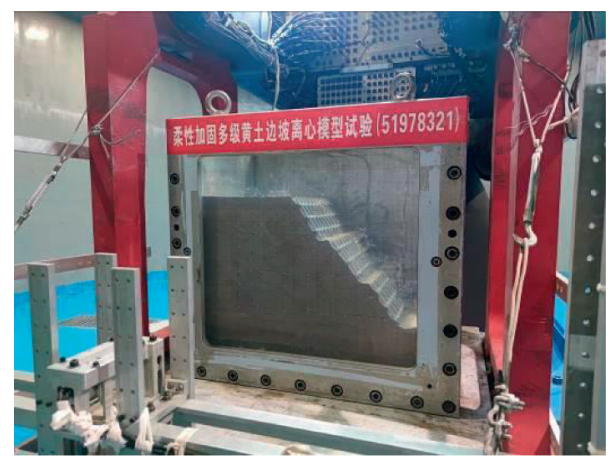

Figure 7: Model slope after placement.

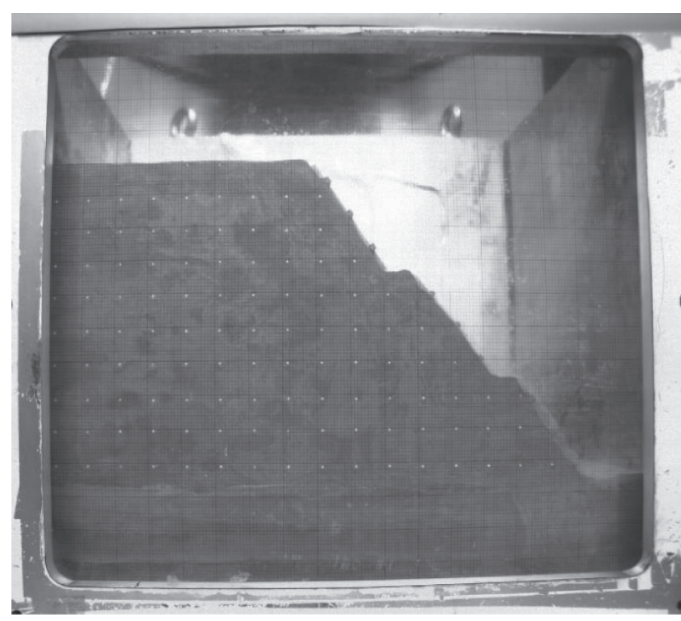

(a)

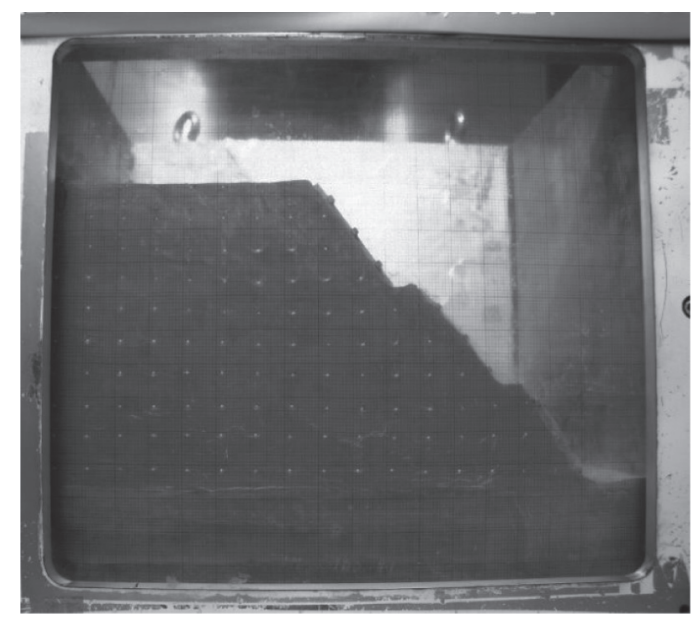

(b)

Figure 8: High-resolution images taken by the centrifuge. (a) High-resolution image at the beginning of the test. (b) High-resolution image at an acceleration of $80 \mathrm{~g}$.

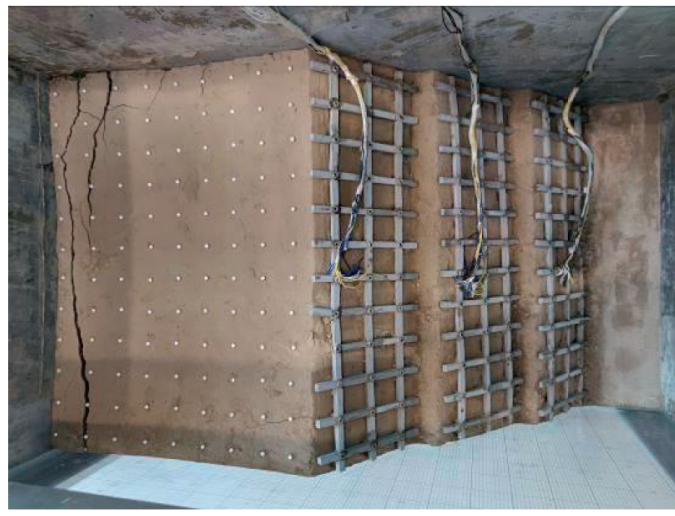

(a)

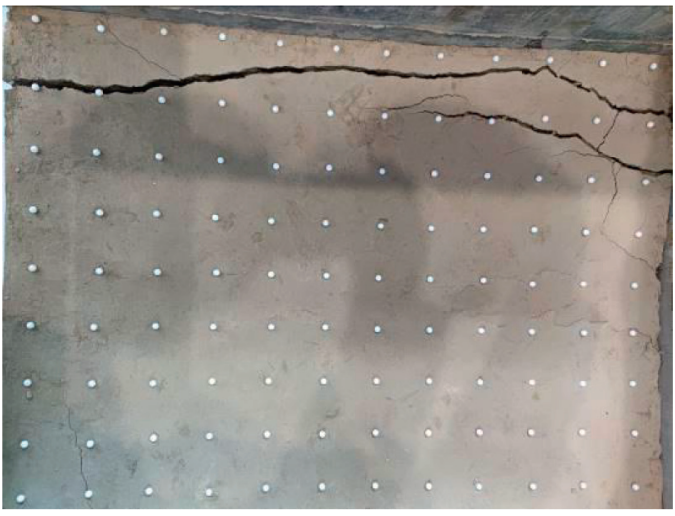

(b)

Figure 9: Slope deformation after test. (a) Overall deformation diagram of slope. (b) Partial diagram of slope top crack.

Under high centrifugal acceleration, the positions of the grid lines in the high-resolution images are different at different times. It is more reasonable to take the intersection of grid lines as the reference point when positioning, and the displacement obtained is also more accurate. According to this method, the slope displacement vector obtained by comparing the slope displacement monitoring points before and after deformation is shown in Figure 10.

It can be seen from Figures 8 to 10 that the deformation of the slope is obvious under high centrifugal acceleration, the vertical displacement decreases gradually from the top of the slope to the bottom, the horizontal displacement is 


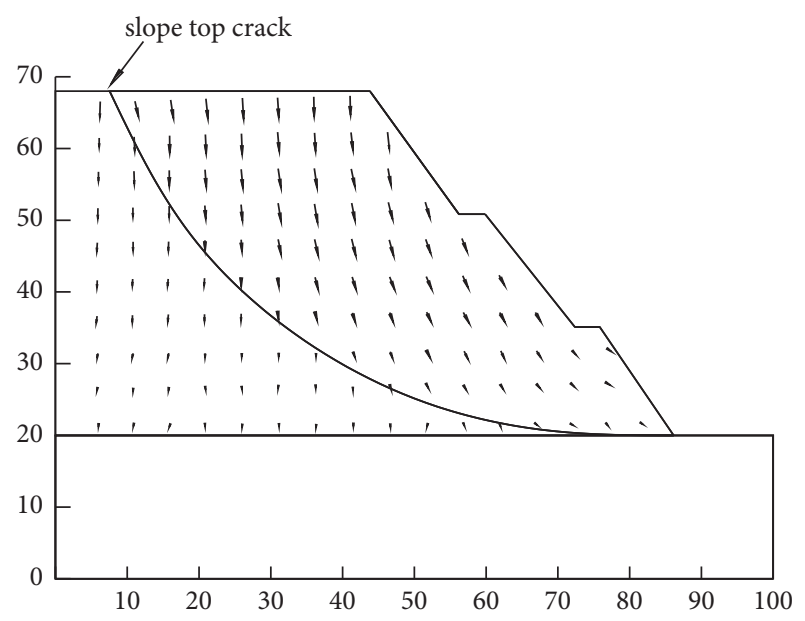

Figure 10: Slope deformation vector diagram (unit: $\mathrm{mm}$ ).

mainly located near the slope surface side, and the maximum horizontal displacement of the slope appears at the toe of the second grade slope. If the area with horizontal displacement is regarded as the sliding area, the position of the sliding surface of the slope can be obtained, which extends from the crack on the slope top down along the end of the anchoring section to the bottom of the slope. This shows that the sliding deformation zone basically coincides with the reinforcement range of the anchor rod, and the frame anchor supporting structure can effectively mobilize the soil deformation in a wider range. At the same time, there is only vertical consolidation settlement in the depth of the slope, and the deformation difference between the deep soil of the slope and the shallow soil near the slope surface side is the main reason for the uneven settlement of the slope top and the cracks at the rear edge of the slope top.

Furthermore, it can be seen from the horizontal displacement distribution of the reference points of each horizontal row that the maximum horizontal displacement of the slope does not appear at the slope surface but in the middle of the deformation zone. The analysis shows that this is mainly related to the constraints of the frame structure on the slope displacement. When the lateral displacement of the soil near the slope surface side occurs, there is a large Earth pressure between the soil and the frame structure. However, this part of the Earth's pressure cannot be released, which will cause the soil in the shallow range near the slope surface side to show compression characteristics.

\subsection{Analysis of Earth Pressure during Loading}

3.2.1. Change of Earth Pressure in Slope with Acceleration. The self-weight loss of the model slope under centrifugal loading can be compensated. The Earth pressure measured in the test is consistent with the actual value of the corresponding point of the prototype slope, so the measured test value can reflect the actual stress state of the slope. The changes in the horizontal Earth pressure of each measuring point in the slope with acceleration are shown in Figure 11.

It can be seen from Figure 11 that the closer the Earth pressure sensor to the bottom of the slope on the same

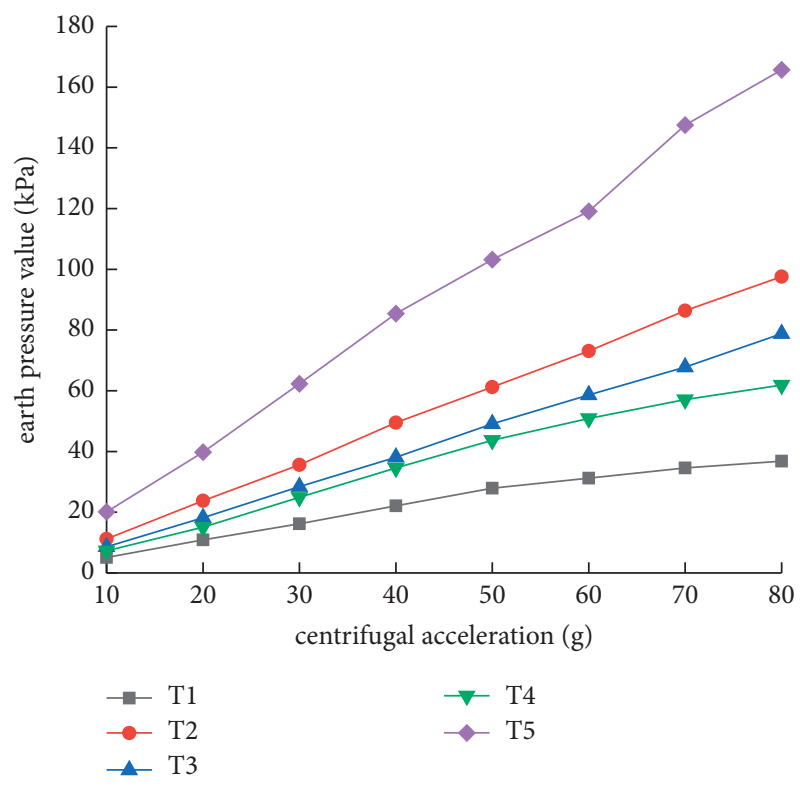

Figure 11: Variation curve of earth pressure in slope with acceleration.

vertical plane, the greater the stress value, that is, $\mathrm{T} 5>\mathrm{T} 3>\mathrm{T} 1$, and the closer the Earth pressure sensor to the slope surface on the same horizontal plane, the smaller the stress value, that is, T4 $<\mathrm{T} 3<\mathrm{T} 2$. At the same time, the Earth pressure value of each measuring point increases with the increase of acceleration, but the growth rate is slightly different. T2, T3, and T5 outside the sliding area basically increase linearly with the increase of acceleration. T1 and T4 inside the sliding area show the same growth trend as the other three measuring points in the initial stage, and the growth rate slows down in the final stage. The analysis believes that this is related to the deformation of the slope. When the acceleration is small, the deformation of the slope is small, and there is no obvious horizontal displacement. At this stage, the growth rate of Earth pressure at each measuring point with acceleration remains basically unchanged. When the acceleration is large, the deformation of the slope increases, the downward sliding trend of soil is obvious, and the horizontal displacement is gradually visible. At this time, the deformation can release some soil pressure, so the growth rate of Earth pressure in the sliding area slows down.

3.2.2. Distribution of Earth Pressure on Frame. The Earth pressure sensor arranged under the frame column can measure the Earth pressure on the frame under different accelerations. The obtained Earth pressure distribution on the frame is shown in Figure 12, in which T6, T7, and T8 correspond to the measuring points of the highest level, middle level, and lowest level frames, respectively.

It can be seen from Figure 12 that the Earth pressure value of each measuring point increases with the increase of acceleration, which shows that the downward sliding trend of the slope is more and more obvious. At the same time, comparing the relative values of each measuring point, it can be found that the Earth pressure from the top of the slope to 


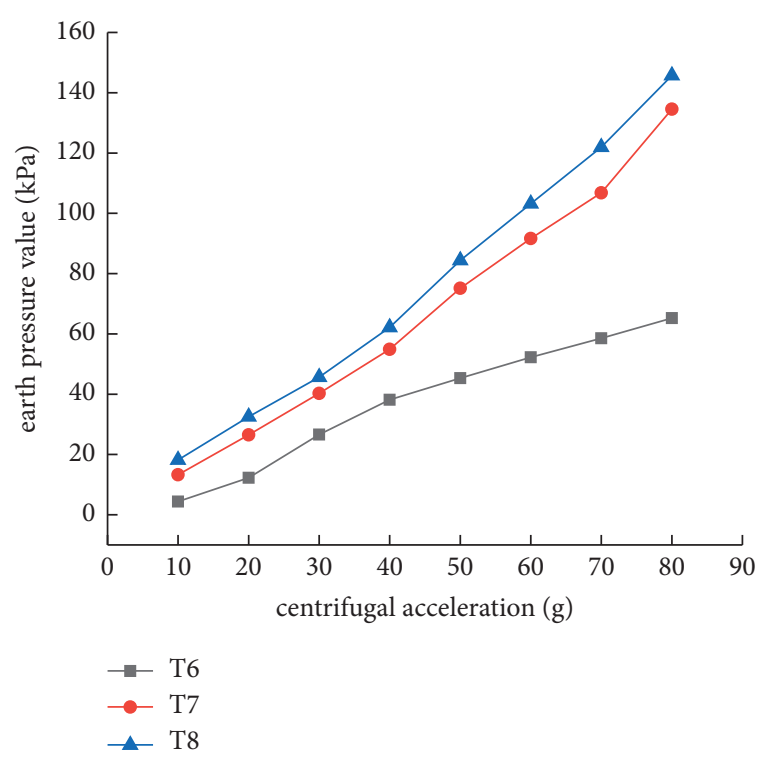

FIGURE 12: Variation curve of Earth pressure on the frame with acceleration.

the bottom is increasing, but the overall trend is not strictly triangular. The Earth pressure of the middle frame increases significantly compared with the top frame, but the growth of the bottom frame is smaller than that of the middle frame. The analysis shows that the Earth pressure under the frame column is related to the deformation of the slope and the position of the anchor rod. In the first grade slope, the soil mainly undergoes vertical settlement, and the horizontal displacement is small, so the Earth pressure acting on the frame is relatively small. In the third grade slope, the anchoring section of the anchor rod goes deep into the stable soil layer, which can provide a large anchoring force and the corresponding Earth pressure is the largest. In the second grade slope, the deformation at the slope toe is the largest, but the anchoring section is basically within the deformation zone, so the Earth pressure on the frame of this grade is between the two.

3.3. Stress Analysis of Anchor during Loading. Axial force is the most important mechanical index to evaluate the anchoring effect of anchor rods, and its analysis is helpful to understand the working principle of supporting structure. In the test, the axial force of the anchor rod is determined according to the axial strain of the anchoring section, and the axial strain is calculated by the paired microstrain values of each measuring point according to the following formula. The axial strain distribution of the test bolt is shown in Figure 13.

$$
\varepsilon=\frac{\left(\varepsilon_{1}+\varepsilon_{2}\right)}{2},
$$

where $\varepsilon$ is axial microstrain; $\varepsilon_{1}$ is the measured value of strain gauge above the measuring point; and $\varepsilon_{2}$ is the measured value of the strain gauge below the measuring point.

After comprehensively comparing the distribution and variation law of the axial force of the three test anchor rods, it can be seen that the axial strain is distributed in a convex shape along the anchoring section. The axial strain growth rate at the end of the anchoring section is greater than that in the middle, and the axial strain at the front end of the anchoring section is lower than that in the middle. This shows that the side friction at the end of the anchoring section is the first to play, while there is reverse side friction at the front end of the anchoring section opposite to the tensile force.

At the same time, the maximum axial strain of the anchor rod in different loading stages appears at different monitoring points. When the acceleration is small, the maximum axial strain of the anchor rod appears at the second measuring point. With the increasing acceleration, the maximum axial strain gradually transits from the second measuring point to the fourth measuring point. This again shows that the axial force of the anchor rod starts at the end of the anchoring section and continues to develop outward. Further combined with the deformation of the slope and the axial force distribution of the anchor rod, it can be found that the side friction of the anchor rod is related to the relative displacement between the anchor rod and the soil. There is compression deformation in the soil within a certain depth under the slope surface, and the soil moves to the inner side of the slope relative to the anchor rod, which leads to the generation of reverse side friction around the anchor rod and the gradual reduction of the axial force at the front end of the anchoring section. However, in the end and middle of the anchoring section, the anchor rod has a tendency to shift toward the slope surface relative to the surrounding soil under the action of tensile force, so the axial force of this section gradually increases along the anchor rod toward the slope surface.

It can be seen from the further comparison that the axial force of the anchor rod at different positions under the same centrifugal acceleration is not the same. Therefore, in order to analyze the relative magnitude of the axial force of the anchor rods at different positions, take the axial force at the end of each test rod for comparison, as shown in Figure 14. The abscissa 1, 2, and 3 correspond to the test anchor rods of the highest, middle, and lowest slopes, respectively.

It can be seen from Figure 14 that the axial force at the end of the $2 \#$ anchor rod is the largest, followed by the $3 \#$ anchor rod, and the axial force at the end of the $1 \#$ anchor rod is the smallest. The analysis shows that the axial force of the anchor rod is not only related to the relative displacement between the anchor rod and soil but also affected by slope deformation and Earth pressure. In the first grade slope, although the vertical displacement of the slope is the largest, the Earth pressure is relatively small, and soil will drive the anchor rod to move downward together. The deformation of the anchoring section is more synchronous with the soil, the side friction is relatively small, and the axial force is small. In the second grade slope, the lateral displacement and Earth pressure are both large, and the middle and rear section of the anchor rods have an outward displacement trend relative to the surrounding soil, the side friction has been effectively brought into play, and the axial force is relatively large. In the third grade slope, the reason 


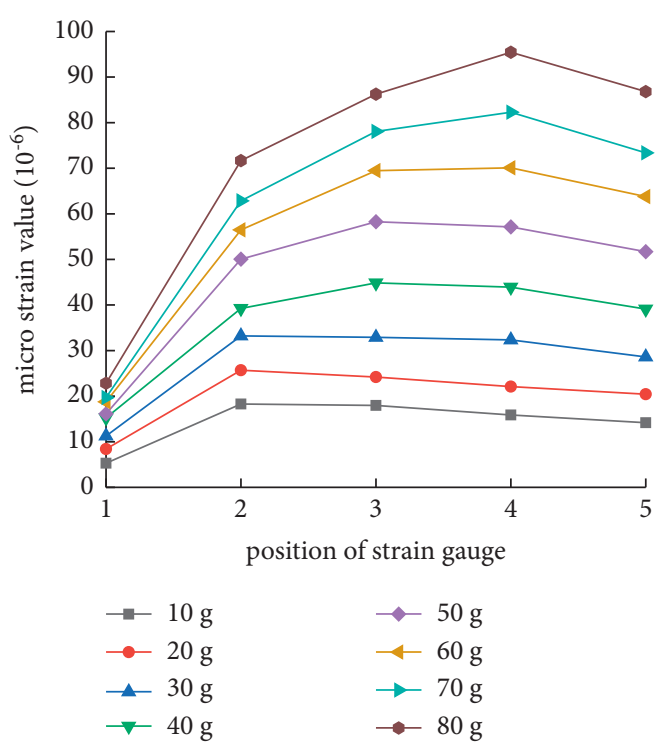

(a)

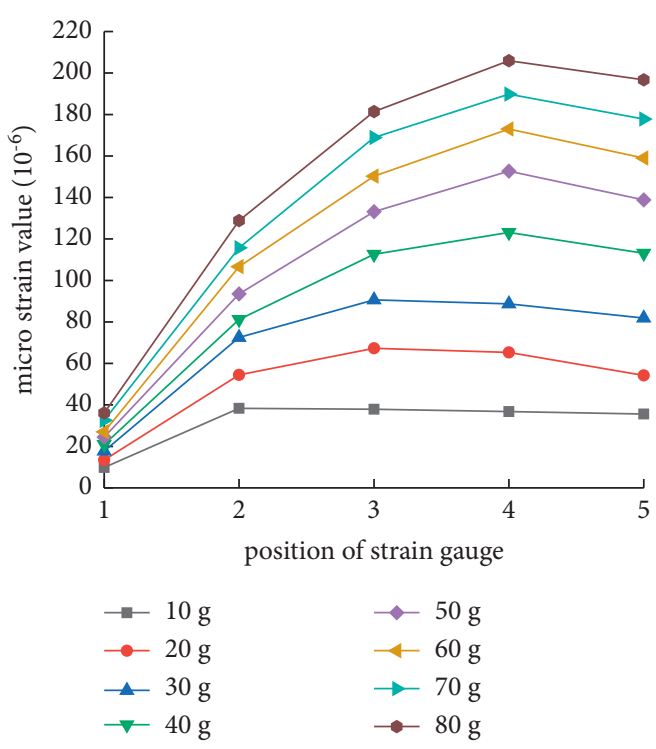

(b)

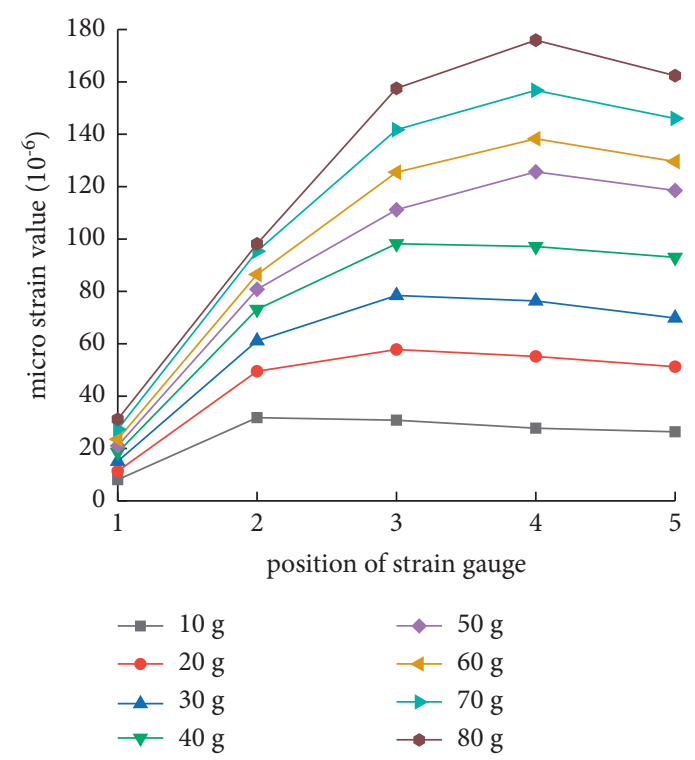

(c)

FIGURE 13: Axial strain distribution of test anchor rod. (a) 1\# anchor rod. (b) 2\# anchor rod. (c) 3\# anchor rod.

why the axial force of $3 \#$ anchor rod is smaller than that of 2\# anchor rod is mainly related to the selection of the test anchor rod. The anchor bolt at the lowest layer of the slope is anchored in sandstone, which can provide greater anchoring force, so $3 \#$ anchor bolt needs to share less anchoring force.

\section{Discussion and Suggestions}

4.1. Comparison of Stress and Deformation Characteristics. It can be seen from the centrifugal test that the sliding surface of the multistage loess slope supported by frame structure with anchors is located in the deep part of the slope, and the sliding deformation range of the slope and the stress characteristics of the anchor rod are different from other types of slopes supported by frame structure with anchors. Therefore, it is necessary to compare the slope deformation and anchor rod stress obtained from the test with some results in the existing literature.

4.1.1. Difference between Multistage Loess Slope and Slope with Structural Plane under Frame Anchor Support. Usually, engineers and technicians will set the anchoring section of the anchor rod in the stable rock and soil when designing the frame anchor supporting structure because the side friction between the anchoring section and the stable rock and soil can provide a large anchoring force. In the test of frame anchor supporting structure, some scholars made the rock and soil in the sliding zone and stable zone according to different materials or used polyethylene film, dry fine soil, and other materials to construct the structural 


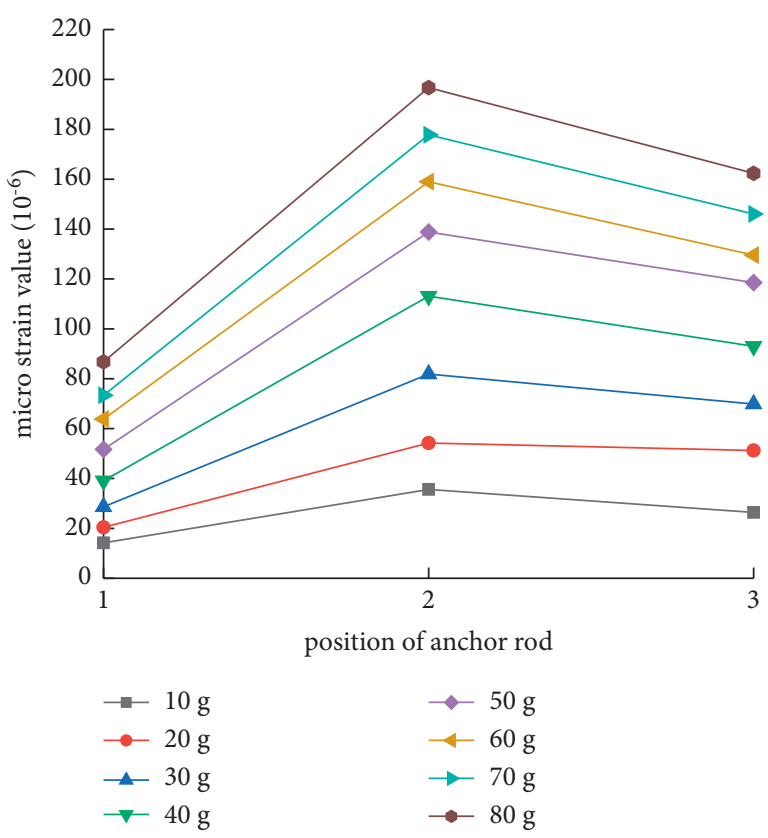

FIGURE 14: Comparison of axial strain at the end of different tested anchor rods.

surface $[17,36]$. The anchoring section can be completely placed in the stable zone by artificially constructing the sliding surface. Obviously, when there is a structural surface or the position of the sliding surface is known, such an experimental design is very scientific. The slope will slide downward along the known sliding surface under external load.

However, different from the above-mentioned rock and soil conditions, the soil layer in the loess area is thick and uniform, and there is no obvious structural surface, so it is impossible to ensure that the anchoring section must be in a stable soil layer. The designer can only place the anchor section behind the potential sliding surface according to the calculated position of the potential sliding surface. However, it can be seen from the test results that the frame anchor supporting structure can coordinate the deformation with the soil and can mobilize the deformation of the soil in a wider range. The failure surface passing through the anchor rod is difficult to form, and the position of the sliding surface can only be transferred to the deep part of the slope. This is consistent with the centrifugal model test of loess slope supported by soil nailing and pile-nail composite [26].

There are differences in stress characteristics between the anchor rods placed in homogeneous soil and the anchor rod placed in absolutely stable rock and soil. The relative displacement between anchor rod and soil in absolutely stable rock and soil is simple. When the unstable soil slides downward, the force acting on the frame is gradually transmitted from outside to inside through the anchor rod. The axial force of the anchor rod at the sliding surface is the largest and decreases gradually to the depth. The relative displacement between anchor rod and soil in homogeneous soil is complex. When the anchor rod moves outward relative to the surrounding soil, the side friction increases the axial force of the anchor rod. When the anchor rod moves inward relative to the surrounding soil, the side friction reduces the axial force of the anchor rod. The maximum axial force occurs at the position where the relative displacement is zero or at the front end of the anchoring section.

4.1.2. Difference between Multistage Loess Slope and SingleStage Loess Slope under Frame Anchor Support. Table 4 lists some specific parameters of single-stage loess slopes supported by frame structure with anchors given by some scholars. It can be seen that when the single-stage loess slope is supported by the frame anchor structure, the length of the anchor rod is relatively long, the ratio of the anchor rod length to slope height is large, and the anchoring section goes deep into the stable soil layer, which has a good effect on improving slope stability.

However, the height of the multistage slope is relatively high. If the anchor rod is designed according to the ratio of the length of the anchor rod to the height of the slope in the single-stage slope, the length of the anchor rod will be very long, which is great waste in the economy. On the other hand, the existence of the unloading platform makes the overall slope of the multistage slope more gentle than that of the single-level slope. It is not necessary to design too long anchor rods, as long as the slope stability can meet the safety requirements. Therefore, when designing the frame anchor structure for multistage loess slope, the length of the anchor rod is usually controlled within a reasonable range. In this way, although the anchoring section does not go deep into the absolutely stable soil layer, it can significantly improve the soil stability in a certain range under the slope surface.

The difference in the ratio between the length of the anchor rod and the height of the slope is bound to make the failure mode and structural stress characteristics of the multistage loess slope different from that of the single-stage loess slope. The centrifugal test results also proved this. The sliding surface of multistage loess slope supported by frame structure with anchors is located at the end of the anchoring section, rather than the intersection of the nonanchored section and the anchored section, so the deformation range of the slope is large. In terms of the stress characteristics of anchor rods, the single-stage loess slope supported by frame structure with anchors is more similar to the slope with structural surface supported by frame structure with anchors, but it is essentially different from the multistage loess slope supported by frame structure with anchors.

4.2. Reinforcement Suggestions. Through the centrifugal test and the above comparison, it can be seen that the sliding surface of multistage loess slope supported by frame structure with anchors is at the end of the anchor rod, and all the anchor rods are within the deformation zone. This seems to be contrary to the idea that the anchoring section is set in the stable soil layer. In fact, this is not the case. Although the longer the anchor rod length, the better the slope stability, it will also increase the support cost. We must find an appropriate balance between safety and economy. Some 
TABLE 4: Main parameters of single-stage loess slope supported by the frame structure with anchors.

\begin{tabular}{lcccccc}
\hline Stratum & $\begin{array}{c}\text { Slope } \\
\text { height }(\mathrm{m})\end{array}$ & $\begin{array}{c}\text { Gradient } \\
\left({ }^{\circ}\right)\end{array}$ & $\begin{array}{c}\text { Horizontal spacing of } \\
\text { anchor rod }(\mathrm{m})\end{array}$ & $\begin{array}{c}\text { Vertical spacing of } \\
\text { anchor rod }(\mathrm{m})\end{array}$ & $\begin{array}{c}\text { Maximum length of } \\
\text { anchor rod (m) }\end{array}$ & $\begin{array}{c}\text { Ratio of maximum length of } \\
\text { anchor rod to slope height }\end{array}$ \\
\hline Li et al. [5] & 10 & $80^{\circ}$ & 2.5 & 2.5 & 17 & 1.70 \\
Lin et al. [37] & 12 & $60^{\circ}$ & 3.0 & 3.0 & 16 & 1.33 \\
Ye et al. [38] & 12 & $80^{\circ}$ & 2.0 & 2.0 & 16 & 1.33 \\
Dong et al. [39] & 11 & $80^{\circ}$ & 2.5 & 2.5 & 15 & 1.36 \\
\hline
\end{tabular}

scholars pointed out from experience that sufficient nonanchored section length and reasonable anchored section length should be ensured in support design, but there is no clear explanation on the specific length of anchor bolt in multistage soil slope [40]. The author believes that the determination of anchor rod length is related to the position of the potential sliding surface, but the anchoring section cannot be designed with the potential sliding surface as the boundary. Because if the anchoring section is placed outside the potential sliding surface of the unsupported slope, the potential sliding surface moving backward after support may not meet the stability requirements. If the anchoring section is always located outside the potential sliding surface of the supported slope by increasing the anchor rod length, the required anchor rod length is too long, which is bound to bring economic waste.

For multistage loess slope, the frame anchor supporting structure and the soil within the reinforcement range can form a whole. When the slope is subjected to an external load, some stable soil before support will undergo coordinated deformation with the supporting structure after support and jointly bear the sliding effect of the slope. Therefore, it is more reasonable to strictly control the end position of the anchorage section from the perspective of changing the position of the potential sliding surface when the frame anchor structure is used to support the multistage loess slope. In this way, the potential sliding surface can be moved to the deep part of the slope through the combination of frame and anchor rod, and then rely on the shear strength of soil and some anchoring force to resist the downward sliding of the slope. At the same time, on the premise that the anchorage depth meets the requirements, the designer should also appropriately increase the length of the nonanchored section and strictly control the length of the anchored section.

\section{Conclusion}

Taking the three-stage loess slope supported by frame structure with anchors as the research object, this paper analyses the structural stress and slope deformation with the help of a large-scale geotechnical centrifugal test and obtains the following conclusions:

(1) The frame anchor supporting structure has a good support effect for multistage loess slope. The deformation between structure and soil can always coordinate with each other, and the failure process has certain toughness characteristics. The amount of deformation in different areas of the slope is different, and the deformation mode of the slope under different acceleration is also different. The sliding deformation zone basically coincides with the reinforcement range of the anchor rods, the transverse crack appears at the rear edge of the slope top, and the sliding surface extends from the crack on the slope top down along the end of the anchoring section to the lowest slope toe.

(2) The deformation of the slope will release part of the soil pressure, the growth rate of the horizontal Earth pressure in the deformation area slows down with the acceleration, and the horizontal Earth pressure in the stability area always increases linearly with the increase of the load. The Earth pressure on the frame gradually increases from the top of the slope to the bottom, but the overall trend is not strictly triangular, and the difference between the Earth pressure on the middle level and the lowest level frame is small.

(3) The axial strain of the anchor rod is convex distributed along the anchoring section, and the maximum axial strain in different loading stages appears at different measuring points. The side friction at the end of the anchoring section is the first to play, and there is reverse side friction at the front end of the anchoring section. The exertion of axial force is not only related to the relative displacement between anchor rod and soil but also affected by slope deformation and Earth pressure. The lowest anchor rod anchored in sandstone can provide greater anchoring force, which makes the axial force of the test anchor rod in the lowest slope less than that in the middle slope.

(4) Compared with the slope with the structural surface, the soil properties of multistage loess slope are more uniform, there is no obvious structural surface, and it is impossible to ensure that the anchor rod must be located in the stable soil layer. Compared with singlelevel loess slope, the height of multistage loess slope is higher, and the ratio of anchor rod length to slope height is smaller. These differences will make the multistage loess slope supported by frame anchor different from the slope with the structural plane and single-stage slope in the stress characteristics and sliding deformation range.

(5) When the frame anchor structure is used to support the multistage loess slope, the anchoring section cannot be designed completely with the potential sliding surface as the boundary. It is more reasonable 
to strictly control the end position of the anchorage section from the perspective of changing the position of the potential sliding surface. This design can make the potential sliding surface move to the deep part of the slope and effectively prevent the slope from sliding downward.

\section{Data Availability}

The data used to support the findings of this study are available from the corresponding author upon request.

\section{Conflicts of Interest}

The authors declare that there are no conflicts of interest regarding the publication of this paper.

\section{Acknowledgments}

This research was financially supported by the National Natural Science Foundation of China (Grant no. 51978321), the Program for Changjiang Scholars and Innovative Research Team in University of Ministry of Education of China (Project no. IRT17R15), the Youth Science and Technology Fund Plan of Gansu Province (Project no. 2020A-031), and the University Innovation Fund Project of Gansu Province (Project no. 20JR5RA434).

\section{References}

[1] Z. X. Yan, S. Zhang, X. Zhang, and P. Jiang, "Dynamic response law of loess slope with different shapes," Advances in Materials Science and Engineering, vol. 2019, Article ID 4853102, 7 pages, 2019.

[2] H. Qiu, A. D. Regmi, P. Cui, M. Cao, J. Lee, and X. Zhu, "Size distribution of loess slides in relation to local slope height within different slope morphologies," Catena, vol. 145, pp. 155-163, 2016.

[3] Y. Zhou, Y. P. Zhu, and S. H. Ye, "Discussion on several issues about design and construction of grillage flexible slope supporting structure with prestressed anchors," Rock and Soil Mechanics, vol. 32, no. S2, pp. 437-443, 2011.

[4] X. F. Huang, X. H. Yang, Y. P. Zhu, and S. J. Zhang, "Comparative analysis of common retaining structures in Xining area," Chinese Journal of Geotechnical Engineering, vol. 34, no. S1, pp. 432-439, 2012.

[5] Z. Li and Y. P. Zhu, "Stability calculation methods for grillage supporting structure with prestressed anchor and its application," Chinese Journal of Rock Mechanics and Engineering, vol. 21, no. 21, pp. 3922-3926, 2005.

[6] Y. P. Zhu and S. H. Ye, "Simplified analysis of slope supported with frame-anchors under lateral seismic loading," Engineering Mechanics, vol. 28, no. 12, pp. 27-32+38, 2011.

[7] J. H. Dong, Y. P. Zhu, W. Ma, and Z. Li, "Simplified seismic design method of frame supporting structure with prestressed anchors for slope stability," China Journal of Highway and Transport, vol. 25, no. 5, pp. 38-46, 2012.

[8] H. Z. Su, J. Hu, and M. Yang, "Evaluation method for slope stability under multianchor support," Natural Hazards Review, vol. 16, no. 4, Article ID 04014034, 2015.

[9] H. B. Xue, F. N. Dang, X. T. Yin, M. Lei, and Q. Deng, "Stability analysis methods for strain-softening slopes,"
Chinese Journal of Geotechnical Engineering, vol. 38, no. 3, pp. 570-576, 2016.

[10] A. M. Han, J. G. Li, J. H. Xiao, and H. Z. Xu, "Mechanical behaviors of frame beam supporting structure with prestressed anchors," Rock and Soil Mechanics, vol. 31, no. 9, pp. 2894-2900, 2010.

[11] Y.-l. Lin, Y.-x. Li, L.-h. Zhao, and T. Y. Yang, "Investigation on seismic response of a three-stage soil slope supported by anchor frame structure," Journal of Central South University, vol. 27, no. 4, pp. 1290-1305, 2020.

[12] Y. L. Lin, L. Lu, and G. L. Yang, "Seismic behavior of a singleform lattice anchoring structure and a combined retaining structure supporting soil slope: a comparison," Environmental Earth Sciences, vol. 79, no. 3, 2020.

[13] Y. R. Zheng, "Development and application of numerical limit analysis for geological materials," Chinese Journal of Rock Mechanics and Engineering, vol. 31, no. 7, pp. 1297-1316, 2012.

[14] Y. P. Zhu, X. Y. Yang, X. R. Ma, X. H. Yang, and S. H. Ye, "Several questions of double reduction method for slope stability analysis," Rock and Soil Mechanics, vol. 39, no. 1, pp. 331-338+348, 2018.

[15] Y. P. Zhu, J. B. Li, S. H. Ye, and Q. Zhang, "Health monitoring and analysis on high slope anchor lattice beam supporting structure," Engineering Mechanics, vol. 32, no. S1, pp. 271-276+283, 2015.

[16] J. B. Hao, J. H. Li, T. Cheng, Y. M. Men, and B. Q. Wang, "Experimental study of slopes supported with framed anchors on shaking table," Chinese Journal of Rock Mechanics and Engineering, vol. 34, no. 2, pp. 293-304, 2015.

[17] J. B. Hao, B. Q. Wang, and N. Li, "Test on failure characteristics of pressure-type anchor with lattice beam under earthquake," China Journal of Highway and Transport, vol. 30, no. 5, pp. 20-27+37, 2017.

[18] D. D. Han, Y. M. Men, and Z. J. Hu, "Experimental study of anti-sliding mechanism and force of lattice anchor in soil landslide," Rock and Soil Mechanics, vol. 41, no. 4, pp. 1189-1194+1202, 2020.

[19] J. J. Zhang, J. Y. Niu, X. Fu, L. C. Cao, and S. J. Yan, "Failure modes of slope stabilized by frame beam with prestressed anchors," European Journal of Environmental and Civil Engineering, 2020.

[20] M. H. Khosravi, J. Takemura, T. Pipatpongsa, and M. Amini, "In-flight excavation of slopes with potential failure planes," Journal of Geotechnical and Geoenvironmental Engineering, vol. 142, no. 5, 2016.

[21] Y. Cheng, A. Huo, Z. Zhao, and J. Peng, "Analysis of loess fracture on slope stability based on centrifugal model tests," Bulletin of Engineering Geology and the Environment, vol. 80, no. 5, pp. 3647-3657, 2021.

[22] Q. S. Meng, L. W. Kong, N. Y. Chen, J. H. Fan, and G. Guo, "Centrifugal model test on slope supporting with pile-anchor combined retaining wall," Rock and Soil Mechanics, vol. 31, no. 11, pp. 3379-3384+3396, 2010.

[23] C. M. Gong, Q. G. Cheng, and Z. P. Liu, "Centrifuge model tests on excavation and reinforcement effect of loess slope," Rock and Soil Mechanics, vol. 31, no. 11, pp. 3481-3486, 2010.

[24] T. B. Li, X. L. Tian, W. X. Han, Y. Ren, Y. He, and Y. X. Wei, "Centrifugal model tests on sliding failure of a pile-stabilized high fill slope," Rock and Soil Mechanics, vol. 34, no. 11, pp. 3061-3070, 2013.

[25] G. Zhang, J. Cao, and L. Wang, "Centrifuge model tests of deformation and failure of nailing-reinforced slope under 
vertical surface loading conditions," Soils and Foundations, vol. 53, no. 1, pp. 117-129, 2013.

[26] Y. F. Wang, Q. G. Cheng, and Y. R. Huang, "Centrifuge tests on excavation of high loess slope with different reinforcement modes," Chinese Journal of Rock Mechanics and Engineering, vol. 33, no. 5, pp. 1032-1046, 2014.

[27] S. Nakamoto, N. Iwasa, and J. Takemura, "Centrifuge modelling of rock bolts with facing plates," International Journal of Physical Modelling in Geotechnics, vol. 18, no. 1, pp. 21-32, 2018.

[28] F. Luo, G. Zhang, Y. Liu, and C. Ma, "Centrifuge modeling of the geotextile reinforced slope subject to drawdown," Geotextiles and Geomembranes, vol. 46, no. 1, pp. 11-21, 2018.

[29] S. Liu, F. Luo, and G. Zhang, "Pile reinforcement behavior and mechanism in a soil slope under drawdown conditions," Bulletin of Engineering Geology and the Environment, vol. 80, no. 5, pp. 4097-4109, 2021.

[30] Z. Y. Cai, H. L. Zhou, G. J. Cai, W. L. Guo, and X. Zhu, "Review of the geotechnical testing and exploration techniques," China Civil Engineering Journal, vol. 53, no. 5, pp. 100-117, 2020.

[31] M. Lin, "Progress of geotechnical centrifuge and specialized test device in China," Journal of Yangtze River Scientific Research Institute, vol. 29, no. 4, pp. 80-84, 2012.

[32] J. Lai, Y. R. Zheng, Y. Liu, X. D. Li, and E. Abi, "Shaking table text study on anti-slide piles and anchor bars of slope under earthquake," China Civil Engineering Journal, vol. 48, no. 9, pp. 96-103, 2015.

[33] T. Zhang, Y. M. Men, S. W. Shi, and X. C. Li, "Comparative model test of anti-slide anchor pile and common anti-slide pile to reinforce loess landslide," Journal of Yangtze River Scientific Research Institute, vol. 34, no. 7, pp. 70-76, 2017.

[34] H. G. Wu, L. F. Pai, T. W. Lai, J. D. Zhang, Y. P Zhang, and Y. R Li, "Study on cooperative performance of pile-anchorreinforced soil combined retaining structure of high fill slopes in mountainous airports," Chinese Journal of Rock Mechanics and Engineering, vol. 38, no. 7, pp. 1498-1511, 2019.

[35] C. T. Wang, H. Wang, Y. F. Zhang, W. M. Qin, and H. Min, "Model test and numerical simulation study on the mechanical characteristics of the anchored slide-resistant pile for stabilizing the colluvial landslide," Rock and Soil Mechanics, vol. 41, no. 10, pp. 3343-3354, 2020.

[36] C. L. Chen, Y. P. Yin, Y. M. Men, and H. N. Liu, "Model test on fully grouted lattice beam anchorage," Chinese Journal of Rock Mechanics and Engineering, vol. 36, no. 4, pp. 881-889, 2017.

[37] W. Lin and J. W. Li, "Numerical simulation tests on lattice beam with prestressed cable," Journal of Earthquake Engineering and Engineering Vibration, vol. 29, no. 4, pp. 109-112, 2009.

[38] S. H. Ye and Y. P. Zhu, "Stability analysis of slope supported by frame with pre-stressed anchors under earthquake," Journal of China Coal Society, vol. 37, no. 12, pp. 1994-1998, 2012.

[39] J. H. Dong, Y. P. Zhu, and W. Ma, "Study on dynamic calculation method for frame supporting structure with prestress anchors," Engineering Mechanics, vol. 30, no. 5, pp. 250-258+264, 2013.

[40] L. K. Cheng, P. Zhang, and F. Wang, "Several mechanical concepts for anchored structures in rock and soil," Chinese Journal of Rock Mechanics and Engineering, vol. 34, no. 4, pp. 668-682, 2015. 